\title{
Effects of magnetic fields on the cosmic-ray ionization of molecular cloud cores
}

\author{
M. Padovani ${ }^{1,2}$ and D. Galli ${ }^{2}$ \\ 1 Institut de Ciències de l'Espai (CSIC-IEEC), Campus UAB, Facultat de Ciències, Torre C5-parell 2 ${ }^{a}, 08193$ Bellaterra, Spain \\ e-mail: padovani@ieec.uab.es \\ 2 INAF - Osservatorio Astrofisico di Arcetri, Largo E. Fermi 5, 50125 Firenze, Italy \\ e-mail: galli@arcetri.astro.it
}

Received 8 March 2011 / Accepted 26 April 2011

\begin{abstract}
Context. Low-energy cosmic rays are the dominant source of ionization for molecular cloud cores. The ionization fraction, in turn, controls the coupling of the magnetic field to the gas and hence the dynamical evolution of the cores.

Aims. The purpose of this work is to compute the attenuation of the cosmic-ray flux rate in a cloud core taking into account magnetic focusing, magnetic mirroring, and all relevant energy loss processes.

Methods. We adopt a standard cloud model characterized by a mass-to-flux ratio supercritical by a factor of $\sim 2$ to describe the density and magnetic field distribution of a low-mass starless core, and we follow the propagation of cosmic rays through the core along flux tubes enclosing different amount of mass. We then extend our analysis to cores with different mass-to-flux ratios.

Results. We find that mirroring always dominates over focusing, implying a reduction of the cosmic-ray ionization rate by a factor of $\sim 2-3$ over most of a solar-mass core with respect to the value in the intercloud medium outside the core. For flux tubes enclosing larger masses the reduction factor is smaller, since the field becomes increasingly uniform at larger radii and lower densities. We also find that the cosmic-ray ionization rate is further reduced in clouds with stronger magnetic field, e.g. by a factor $\sim 4$ for a marginally critical cloud.

Conclusions. The magnetic field threading molecular cloud cores affects the penetration of low-energy cosmic rays and reduces the ionization rate by a factor 3-4 depending on the position inside the core and the magnetization of the core.
\end{abstract}

Key words. cosmic rays - ISM: clouds - ISM: magnetic fields

\section{Introduction}

Cosmic rays (CRs) are a primary source of ionization in interstellar clouds (Hayakawa et al. 1961; Spitzer \& Tomasko 1968), contribute to the heating of the gas (Glassgold \& Langer 1973) and affect the chemistry of dense clouds by removing molecules from the most volatiles ices like CO (Hasegawa \& Herbst 1993). For a review of CR induced chemistry in the interstellar medium (ISM), see Dalgarno (2006). In addition, the observed diffuse gamma-ray emission from the galactic plane is believed to be the result of the decay of neutral pions produced during inelastic collisions of high-energy ( $>1 \mathrm{GeV}$ ) CRs with the ISM (see e.g. Gabici et al. 2007).

$\mathrm{CR}$ protons and heavy nuclei in the range $1 \mathrm{MeV} /$ nucleon-1 $\mathrm{GeV} /$ nucleon and $\mathrm{CR}$ electrons in the energy range $10 \mathrm{keV}-10 \mathrm{MeV}$ (hereafter, low-energy CRs) are of particular interest for studies of interstellar chemistry, as they provide the bulk of the ionization in molecular cloud cores of typical $\mathrm{H}_{2}$ column density $N \approx 10^{22} \mathrm{~cm}^{-2}$. Consequently, low-energy CRs determine the degree of coupling of the magnetic field in the gas and control the dynamical evolution of molecular cloud cores. Values of the ionization fraction in molecular cloud cores determined from the column densities of species sensitive to the electron density extend over about 1-2 orders of magnitude (Caselli et al. 1998). It is unclear whether the inferred CR ionization rates span a comparable range or not, due to the sensitivity of the results to the adopted chemical model (Williams et al. 1998;
Wakelam et al. 2006). Since the scatter in the ionization fraction may, in part, reflect intrinsic variations of the CR flux from core to core, it is important to assess the question of the penetration of CRs in molecular cores.

In a previous study, Padovani et al. (2009, hereafter P09) computed the decrease of the $\mathrm{CR}$ ionization rate as function of column density in a plane-parallel, unmagnetized cloud, considering energy losses due to elastic and non-elastic collisions of CRs with the ambient gas, bremsstrahlung, and pion production. However, interstellar magnetic fields can affect the propagation of CRs by magnetic focusing, mirroring, and diffusion (Skilling \& Strong 1976; Cesarsky \& Völk 1978; Chandran 2000; Padoan \& Scalo 2005). The first two effects are due to the non-uniformity of the large-scale (mean) component of the field, whereas the latter is associated to magnetic field fluctuations on the scale of the Larmor radius of CR particles. The relative importance of these processes in the ISM depends on a number of variables not always well determined (geometry and strength of the magnetic field, nature and characteristics of turbulence, etc.), and is unclear whether they can significantly reduce (or enhance) the $\mathrm{CR}$ ionization of molecular cloud cores. In addition, the damping of small-scale magnetic fluctuations (e.g. Alfvén waves) that affect the propagation of CRs is strongly dependent on the ionization fraction of the medium, which, in turn, is mostly determined by the CRs themselves. The general problem must then be addressed in a self-consistent way. 
In this paper we consider the propagation of CRs along the magnetic field lines threading a molecular core assuming that only the mean field affects the CR properties through a combination of magnetic focusing and mirroring. A similar problem was addressed by Desch et al. (2004) to determine the efficiency of spallation reactions and trapping of CR nuclei in the protosolar nebula. In their study, Desch et al. (2004) computed an energy dependent correction factor for the CR flux, representing the fraction of the core's column density a CR of energy $E$ interacts with as it passes through the core or it is thermalized. Our approach is similar, but we extend and refine their method, incorporating the magnetic effects in the propagation model of $\mathrm{P} 09$, that takes into account energy losses, and adopting a specific profile for the density and magnetic field of the core.

This paper is organized as follows: in Sect. 2 we discuss the effects of the magnetic field on the CR propagation; in Sect. 3 we describe the adopted cloud core model; in Sect. 4 we compute the modifications of the CR ionization rate due to the magnetic field; in Sect. 5 we explore the dependence of the results on the mass and magnetization of the core; finally, in Sect. 6 we summarize our conclusions.

\section{Effects of magnetic fields on CR propagation}

CRs are charged particles that perform helicoidal trajectories around the magnetic field lines of the medium where they propagate. For a particle of charge $Z e$ and mass $m$ in an uniform magnetic field, this motion consists of the combination of a circular motion around the field line with velocity $v_{\perp}$ and a uniform motion along the field line with velocity $v_{\|}$. The frequency of the circular motion is the cyclotron frequency, $\Omega_{\mathrm{c}}=Z e B / \gamma m c$. The Larmor radius $r_{\mathrm{L}}$ is given by

$r_{\mathrm{L}}=\frac{v_{\perp}}{\left|\Omega_{\mathrm{c}}\right|}=\frac{p c}{Z e B} \sin \alpha$,

where $p=\gamma m v$ is the particle's momentum and $\alpha$ is the pitch angle (i.e. the angle between the particle's velocity and the magnetic field). The helical step $\delta$, that is the projection along the direction of the magnetic field of the pathlength of the CR during a single rotation around the field lines, is given by

$\delta=v_{\|} \frac{2 \pi}{\left|\Omega_{\mathrm{c}}\right|}=2 \pi \frac{p c}{Z e B} \cos \alpha$.

For a cloud with a magnetic field $B=10 \mu \mathrm{G}$, the Larmor radii of ionizing CRs (CR protons and heavy nuclei with $E \lesssim$ $1 \mathrm{GeV} /$ nucleon and CR electrons with $E \lesssim 10 \mathrm{MeV}$, see P09) are less than $\sim 10^{-7}$ pc and $\sim 10^{-9}$ pc for protons and electrons, respectively, many orders of magnitude smaller than the typical size of Bok globules $(\sim 0.05 \mathrm{pc})$, dense cores $(\sim 1-5 \mathrm{pc})$, and giant molecular clouds ( $\sim 25 \mathrm{pc})$. In the absence of small-scale perturbations in the field, we can therefore assume that low-energy CRs propagate closely following the magnetic field lines.

Perturbations in the forms of magnetohydrodynamic (MHD) waves with wavelength of the order of the Larmor radius of the particle can efficiently scatter CRs. The waves can be part of an MHD turbulent cascade, or can be self-generated by the CRs themselves (Kulsrud 2005). However, in a mostly neutral ISM, turbulent MHD cascades are quenched at scales of roughly the collision mean free path of ions with neutrals, if the ion-neutral collision rate exceeds the energy injection rate. Alfvén waves with wavelength $\lambda<\lambda_{\mathrm{cr}}=\pi v_{\mathrm{A}} /\left(\gamma_{\mathrm{in}} \rho_{i}\right)$, where $v_{\mathrm{A}}$ is the Alfvén speed in the neutrals and $\gamma_{\text {in }}$ the collisional drag coefficient, are efficiently damped by collisions with neutrals (Kulsrud \& Pearce 1969). For typical molecular cloud conditions $\left(n=10^{4} \mathrm{~cm}^{-3}, B=10 \mu \mathrm{G}\right.$, and ionization fraction $\sim 10^{-7}$ ), the critical wavelength is $\lambda_{\mathrm{cr}} \approx 3 \times 10^{-3} \mathrm{pc}$, assuming $\gamma_{\text {in }} \approx 10^{14} \mathrm{~cm}^{3} \mathrm{~s}^{-1} \mathrm{~g}^{-1}$ (Pinto et al. 2008). As we have seen, the Larmor radii of ionizing CR particles are much smaller than the cloud's size or the critical wavelength. Thus, for typical values of the cloud's parameters, only CR particles with energy larger than a few $\mathrm{TeV}$, and Larmor radii $r_{\mathrm{L}} \geq \lambda_{\text {cr }}$ find MHD waves to resonate with. These particles however do not contribute significantly to the ionization of the cloud.

The origin and maintenance of MHD disturbances at the scale of the Larmor radius is unclear: small-scale MHD waves can be self-generated by CRs streaming in the intercloud medium (ICM) or in the cloud itself. Cesarsky \& Völk (1978) have shown that the streaming instability operates in the ICM only for CRs with energy below $\sim 40 \mathrm{MeV} /$ nucleon (for a region of size $1 \mathrm{pc}$, density $10^{3} \mathrm{~cm}^{-3}$, and magnetic field $10 \mu \mathrm{G}$ ) and at even lower energies in the regions surrounding molecular cloud cores. Thus, we expect that $\mathrm{CRs}$ in the energy range between $100 \mathrm{MeV} /$ nucleon and $1 \mathrm{GeV} /$ nucleon, that provide the bulk of the ionization in a cloud core, stream freely through the core without self-generating MHD waves. We ignore therefore the presence of self-generated waves in cloud cores for the rest of this paper, but we believe that this problem deserves further scrutiny.

\subsection{Magnetic focusing and mirroring}

Theoretical models predict that collapsing cloud cores must overcome the support provided by their magnetic field in order to form stars. In the process, the competition between gravity pulling inward and magnetic pressure pushing outward is expected to produce a warped, hourglass pattern of the magnetic field. Recently, this scenario has received support from observations. Maps of polarized dust emission have revealed that the magnetic field in molecular clouds is rather uniform, except near cores where the field becomes strongly pinched and almost radial (see, e.g., Tang et al. 2009). On the other hand highresolution interferometric observations of submillimeter polarized emission in the low-mass core NGC 1333 IRAS 4A by Girart et al. (2006) show a magnetic field geometry consistent with the predictions of theoretical models for the formation of solar-type stars, in which ordered large-scale magnetic fields control the evolution and collapse of molecular cloud cores (see a comparison of observations with theoretical collapse models in Gonçalves et al. 2008). We therefore adopt the hourglass geometry as the basis of our analysis of CR penetration into a cloud core (see Sect. 3).

The effects of magnetic mirroring and focusing in a hourglass geometry can be simply described following e.g. Desch et al. (2004). A charged particle traveling in a magnetized medium conserves its kinetic energy $\gamma m c^{2}$ and its magnetic moment $\mu=\gamma m v^{2} \sin ^{2} \alpha / 2 B$. It follows that CRs propagating from the ICM to the cloud's interior must increase $v_{\perp}^{2}$ to conserve $\mu$ and decrease $v_{\|}$to conserve $|v|^{2}$. Thus, the pitch angle of the particle must increase from the value $\alpha_{\mathrm{ICM}}$ to a value $\alpha$ as

$\frac{\sin ^{2} \alpha}{\sin ^{2} \alpha_{\mathrm{ICM}}}=\frac{B}{B_{\mathrm{ICM}}} \equiv \chi$,

where $\chi>1$. Therefore, a CR starting in the ICM with a pitch angle $\sin \alpha_{\mathrm{ICM}}>1 / \chi^{1 / 2}$ cannot penetrate a region with magnetic field $B>\chi B_{\mathrm{ICM}}$, and will be bounced out (magnetic mirroring). Conversely, the CR flux $j(E)$ in the cloud is increased by 


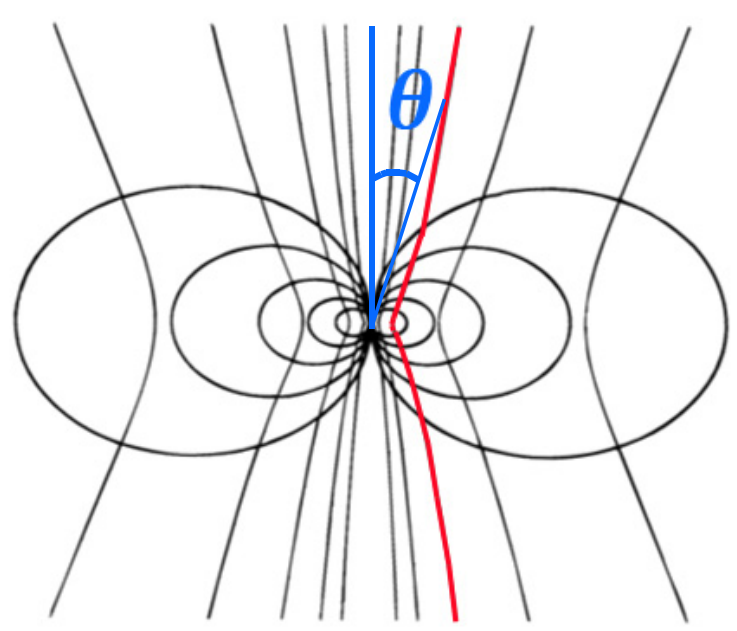

Fig. 1. Isodensity contours (thick curves) and magnetic field lines (thin curves) in the meridional plane of a model with $\lambda=2.66\left(\lambda_{\mathrm{r}}=1.94\right)$, from Li \& Shu (1996). We consider the motion of CRs along a particular field line (red line) as function of the polar angle $\theta$.

the opening out of the field lines by a factor proportional to the density of magnetic field lines per unit area (magnetic focusing),

$$
j(E)=\chi j_{\mathrm{ICM}}(E)
$$

The effects of focusing and mirroring depend only on the magnetic field strength, and are the same for CR protons, electrons, and heavy nuclei.

\section{The cloud model}

In order to study the effects of the magnetic field on the propagation of CRs in molecular cores, we adopt the models of Li \& Shu (1996) (see also Galli et al. 1999), for magnetostatic, scale-free, self-gravitating clouds supported by axiallysymmetric hourglass-like magnetic fields (see Fig. 1). For simplicity, we consider models with an isothermal equation of state. These models are characterized by a value of the nondimensional mass-to-flux ratio $\lambda$ defined by

$\lambda=2 \pi G^{1 / 2} \frac{M(\Phi)}{\Phi}$

where $G$ is the gravitational constant, $\Phi$ the magnetic flux, and $M(\Phi)$ the mass contained in the flux tube $\Phi$. A related quantity is the spherical mass-to-flux ratio $\lambda_{\mathrm{r}}<\lambda$, evaluated from Eq. (5) but considering only the mass enclosed in a sphere tangent to the "waist" of the flux tube. The latter quantity is more appropriate for comparisons with observations, because of the limited beam of a telescope.

The magnetic field of the model is axially symmetric and purely poloidal. In spherical coordinates $(r, \theta)$, it is given by

$\boldsymbol{B}=\frac{1}{2 \pi} \nabla \times\left(\frac{\Phi}{r \sin \theta} \hat{\boldsymbol{e}}_{\phi}\right)$

where

$\Phi(r, \theta)=\frac{4 \pi c_{\mathrm{s}}^{2} r}{G^{1 / 2}} \phi(\theta)$

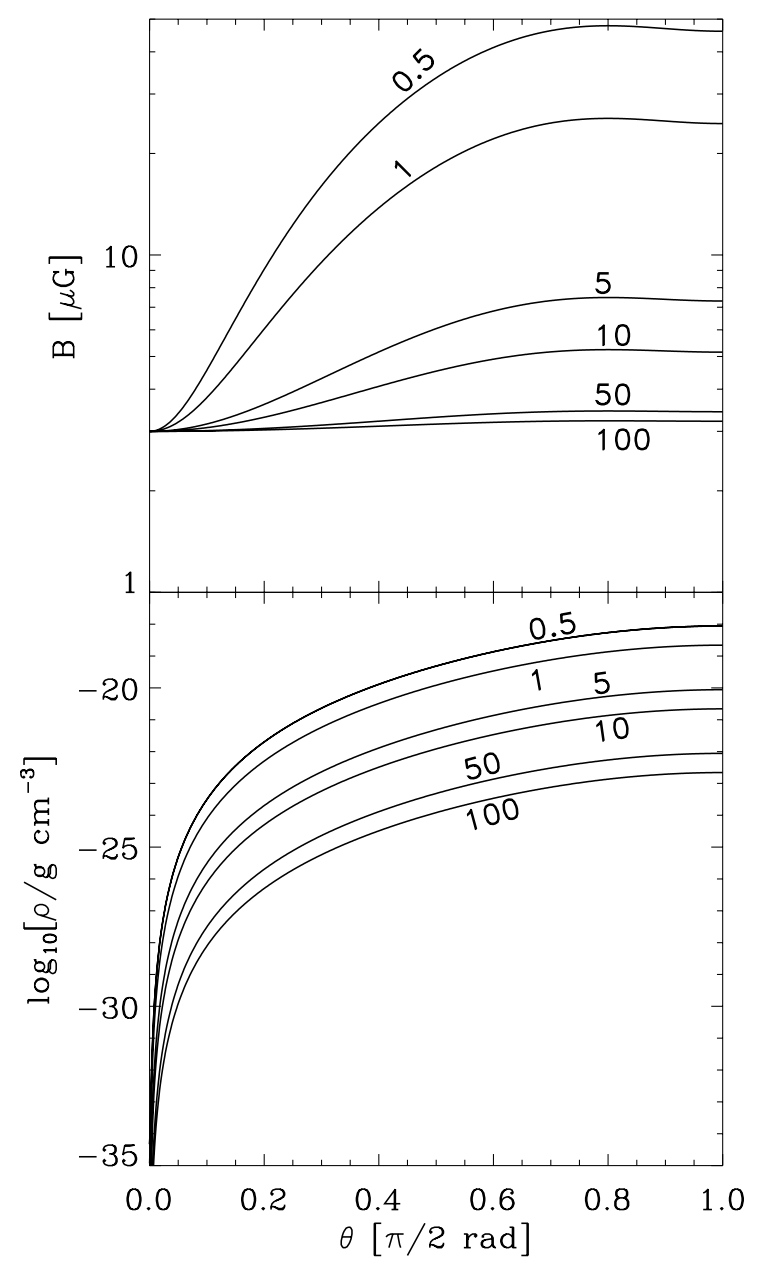

Fig. 2. Modulus of the magnetic field (upper panel) and density (lower panel) as function of the polar angle $\theta$ for flux tubes enclosing different masses (label values in $M_{\odot}$ ).

$c_{\mathrm{S}}$ is the sound speed, and $\phi(\theta)$ is a dimensionless function. Similarly, the density is given by

$\rho(r, \theta)=\frac{c_{\mathrm{s}}^{2}}{2 \pi G r^{2}} R(\theta)$,

where $R(\theta)$ is a dimensionless function. From Eqs. (6) and (7), the radial and polar components of the magnetic field are respectively

$B_{\mathrm{r}}=\frac{2 c_{\mathrm{s}}^{2}}{G^{1 / 2} r \sin \theta} \phi^{\prime} \quad$ and $\quad B_{\theta}=-\frac{2 c_{\mathrm{s}}^{2}}{G^{1 / 2} r \sin \theta} \phi$,

where $\phi^{\prime}=\mathrm{d} \phi / \mathrm{d} \theta$. The modulus of the magnetic field vector is

$|\boldsymbol{B}|=\frac{4 \lambda c_{\mathrm{s}}^{4} \phi^{2}}{G^{3 / 2} M(\Phi) \sin \theta} \sqrt{1+\left(\frac{\phi^{\prime}}{\phi}\right)^{2}}+B_{\mathrm{ICM}}$,

where $B_{\mathrm{ICM}}$ is the intercloud magnetic field, assumed uniform and equal in strength to the Galactic magnetic field.

For our reference model we choose $\lambda=2.66\left(\lambda_{\mathrm{r}}=1.94\right)$, and we assume $c_{\mathrm{s}}=0.2 \mathrm{~km} \mathrm{~s}^{-1}, B_{\mathrm{ICM}}=3 \mu \mathrm{G}$. Figure 2 shows the profiles of the magnetic field strength and the density as function of the polar angle, moving along flux tubes enclosing various masses. In the following we will focus on a flux tube enclosing a mass $M(\Phi)=1 M_{\odot}$, a typical value for a low-mass core, corresponding to an equatorial radius at the "waist" of the flux tube $r_{\mathrm{eq}}=0.036 \mathrm{pc}$ (other cases will be considered in Sect. 5). 


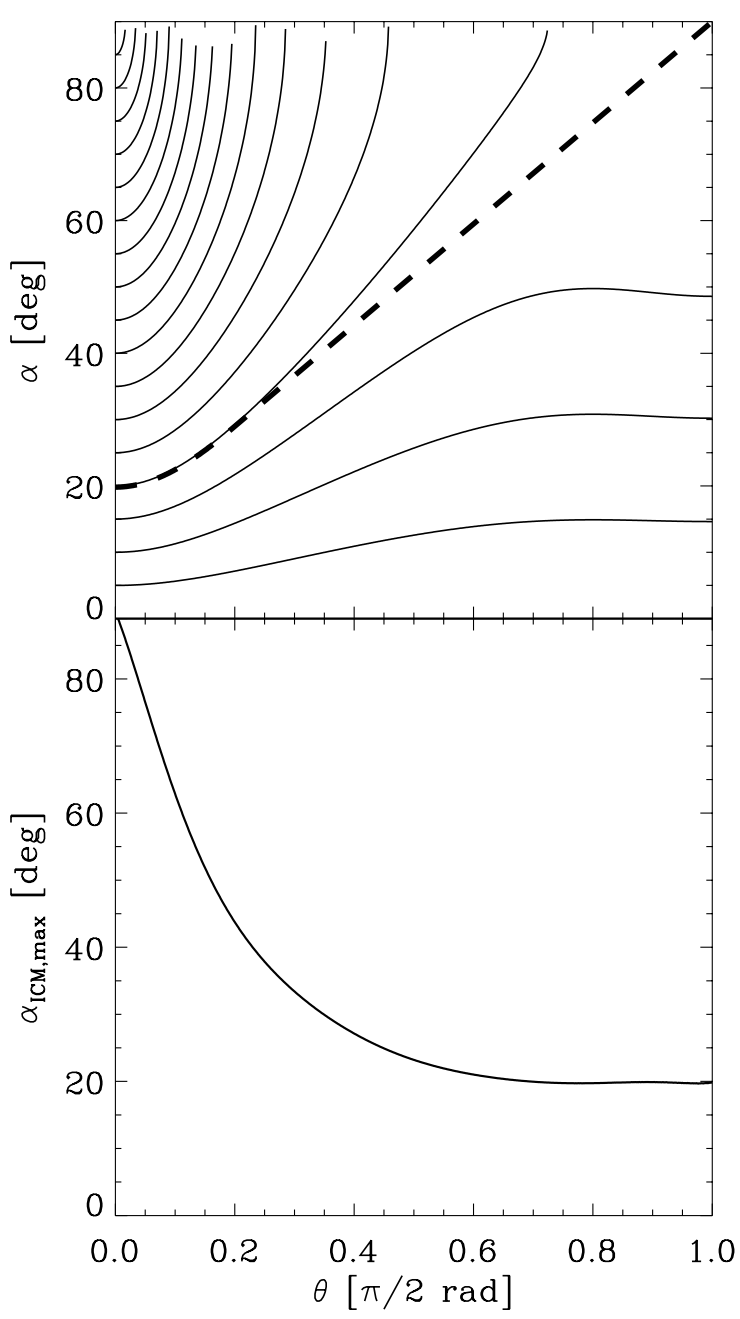

Fig. 3. Upper panel: variation of the CR pitch angle $\alpha$ as function of the polar angle $\theta$ for values of $\alpha_{\mathrm{ICM}}$ between $0^{\circ}$ and $90^{\circ}$ in steps of $5^{\circ}$ (from bottom to top); the dashed line represents the critical pitch angle $\alpha_{\mathrm{cr}}=20.5^{\circ}$. Lower panel: relation between the maximum initial pitch angle $\alpha_{\mathrm{ICM}, \max }$ and the polar angle that can be reached during the propagation inside the core.

The upper panel of Fig. 3 shows the variation of the pitch angle $\alpha$ computed from Eq. (3) as function of the polar angle $\theta$ for various inital values of $\alpha_{\text {ICM }}$ assuming the magnetic field profile of the reference flux tube (see Fig. 2). In this case, only particles starting with pitch angle $\alpha_{\mathrm{ICM}}<\alpha_{\mathrm{cr}}=20.5^{\circ}$ are able to reach the cloud's midplane $(\theta=\pi / 2$, where $\chi=8.174)$. CRs starting with pitch angles larger than this value will be pushed back by magnetic mirroring before reaching the midplane at a position $\theta_{\max }\left(\alpha_{\mathrm{ICM}}\right)<\pi / 2$. Inverting this relation, one finds the value of the maximum allowed pitch angle $\alpha_{\mathrm{ICM}, \max }(\theta)$ for a CR to reach a given position $\theta$, shown in the lower panel of Fig. 3 .

\section{CR ionization rate}

Consider first CRs entering the core from above (in the following denoted by a subscript + ). Only those with pitch angle $\alpha_{\mathrm{ICM}}<$ $\alpha_{\text {ICM,max }}(\theta)$ can reach a position $\theta$ inside the cloud. Thus, their contribution to the $\mathrm{CR}$ ionization rate of $\mathrm{H}_{2}$ is

$$
\begin{aligned}
\zeta_{+}^{\mathrm{H}_{2}}(\theta)= & 2 \pi \chi(\theta) \int_{0}^{\infty} \mathrm{d} E \int_{0}^{\alpha_{\mathrm{ICM}, \max }(\theta)} j\left[E, N\left(\theta, \alpha_{\mathrm{ICM}}\right)\right] \\
& \times[1+\phi(E)] \sigma^{\mathrm{ion}}(E) \sin \alpha_{\mathrm{ICM}} \mathrm{d} \alpha_{\mathrm{ICM}},
\end{aligned}
$$

where $N\left(\theta, \alpha_{\mathrm{ICM}}\right)$ is the column density of $\mathrm{H}_{2}$ into the core (with $N=0$ at $\theta=0), \phi(E)$ is a correction factor accounting for the ionization of $\mathrm{H}_{2}$ by secondary electrons, and $\sigma^{\text {ion }}(E)$ is the ionization cross section of $\mathrm{H}_{2}$. We adopt the continuous-slowingdown approximation to relate the flux of CR particles of energy $E$ at a column density $N$ inside the cloud, $j\left[E, N\left(\theta, \alpha_{\mathrm{ICM}}\right)\right]$, to the flux of CR particles of energy $E_{\mathrm{ICM}}$ in the ICM, $j\left(E_{\mathrm{ICM}}, 0\right)$ :

$j\left[E, N\left(\theta, \alpha_{\mathrm{ICM}}\right)\right]=j\left(E_{\mathrm{ICM}}, 0\right) \frac{L\left(E_{\mathrm{ICM}}\right)}{L(E)}$,

where $L(E)$ is the energy loss function (see P09 for details on the method). The initial and final energies are such that

$N=n\left[R\left(E_{\mathrm{ICM}}\right)-R(E)\right]$,

where $n$ is the local density of $\mathrm{H}_{2}$ and $R(E)$ is the particle's range. We consider CRs composed of electrons, protons, and heavy nuclei. As stressed by Webber (1998) and P09, CR electrons contribute significantly to the total ionization rate, and cannot be neglected. We adopt four combinations of proton/electron spectra $j(E)$, labeled as in P09: Webber (1998, W98) and Moskalenko et al. (2002, M02) for protons and heavy nuclei; Strong et al. (2000, C00 and E00) for electrons. The spectra M02 and E00 are characterized by an increase at low energies, whereas the spectra W98 and C00 are constant or slightly decreasing.

The column density passed through by a CR propagating along a magnetic field line is

$N\left(\theta, \alpha_{\mathrm{ICM}}\right)=\frac{1}{\mu m_{\mathrm{H}}} \int \rho \frac{\mathrm{d} \ell}{\cos \alpha}$,

where $\mu=2.8$ is the molecular weight, $\mathrm{d} \ell=\left[\mathrm{d} r^{2}+(r \mathrm{~d} \theta)^{2}\right]^{1 / 2}$ is the element of magnetic field line, and the factor $1 / \cos \alpha$ accounts for the increase of the actual path length of a CR performing a helicoidal trajectory around a magnetic field with respect to the displacement along the field line. Substituting in Eq. (14) the expressions for $\rho$ and $\mathrm{d} \ell$, of our model, we obtain the actual column density passed through by CRs propagating from the ICM to the mirror point $\theta_{\max }\left(\alpha_{\mathrm{ICM}}\right)$,

$N\left(\theta, \alpha_{\mathrm{ICM}}\right)=\frac{c_{\mathrm{s}}^{4} \lambda}{\pi \mu m_{\mathrm{H}} G^{2} M(\Phi)} \int_{0}^{\theta_{\max }} \frac{\phi(\theta) R(\theta)}{\cos \alpha(\theta)} \sqrt{1+\left(\frac{\phi^{\prime}}{\phi}\right)^{2}} \mathrm{~d} \theta$,

where $\cos \alpha(\theta)$ is given in terms of $\alpha_{\mathrm{ICM}}$ and $\chi$ by Eq. (3).

The results are shown in Fig. 4 for the reference flux tube enclosing $1 M_{\odot}$ and for different values of $\alpha_{\mathrm{ICM}}$. It is evident from the figure that as a first approximation one can assume that CRs coming from the ICM and traveling toward the cloud's midplane experience a similar increase in column density, independently on the initial pitch angle $\alpha_{\mathrm{ICM}}$, the latter mainly determining the value of the column density at which the CRs are pushed out by magnetic mirroring. We will then assume for all CRs the column density profile corresponding to $\alpha_{\mathrm{ICM}}=0$, but truncated at increasingly larger values depending on the initial pitch angle $\alpha_{\mathrm{ICM}}$,

$N\left(\theta, \alpha_{\mathrm{ICM}}\right) \approx N(\theta, 0)$ if $0<\theta<\theta_{\max }\left(\alpha_{\mathrm{ICM}}\right)$.

With this approximation Eq. (11) simplifies to

$$
\begin{aligned}
\zeta_{+}^{\mathrm{H}_{2}}(\theta)= & 2 \pi \chi(\theta) \int_{0}^{\alpha_{\mathrm{ICM}, \max }(\theta)} \sin \alpha_{\mathrm{ICM}} \mathrm{d} \alpha_{\mathrm{ICM}} \\
& \times \int_{0}^{\infty} j\left[E, N_{0}(\theta)\right][1+\phi(E)] \sigma^{\mathrm{ion}}(E) \mathrm{d} E,
\end{aligned}
$$


M. Padovani and D. Galli: Effects of magnetic fields on the cosmic-ray ionization of molecular cloud cores

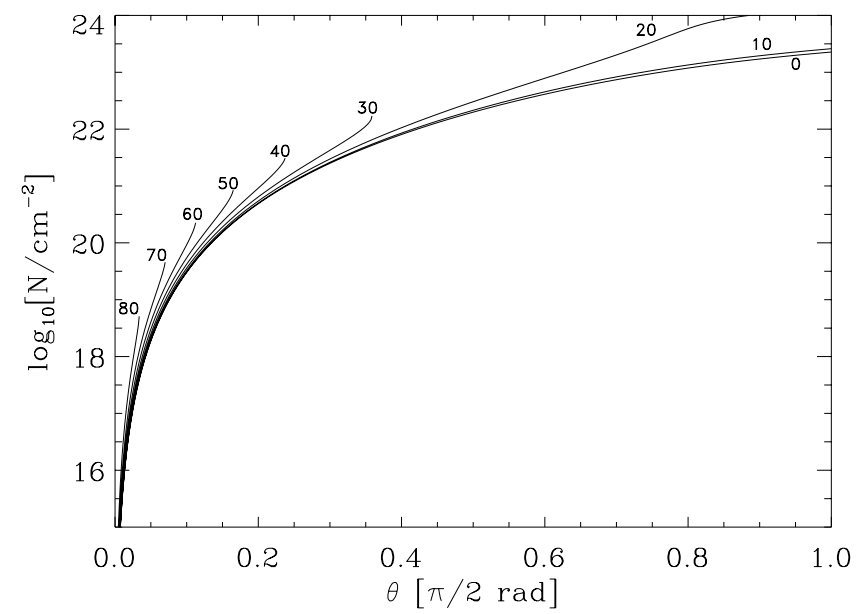

Fig. 4. Column density passed through before reaching the mirror point by CRs propagating along a field line of the reference flux tube enclosing $1 M_{\odot}$, as function of the polar angle $\theta$ for different values of the initial pitch angle $\alpha_{\mathrm{ICM}}$ (labels in degrees).

where, for simplicity, we have defined $N_{0}(\theta)=N(\theta, 0)$ to indicate the column density measured along a field line. The second integral of Eq. (17) is the CR ionization rate computed assuming one-dimensional propagation, calculated in P09:

$\int_{0}^{\infty} j\left[E, N_{0}(\theta)\right][1+\phi(E)] \sigma^{\text {ion. }}(E) \mathrm{d} E \equiv \frac{1}{4 \pi} \zeta_{0}^{\mathrm{H}_{2}}\left[N_{0}(\theta)\right]$.

Thus, Eq. (11) can be rewritten as

$\zeta_{+}^{\mathrm{H}_{2}}(\theta)=\varphi_{+}(\theta) \zeta_{0}^{\mathrm{H}_{2}}\left[N_{0}(\theta)\right]$,

where

$\varphi_{+}(\theta)=\frac{\chi(\theta)}{2}\left[1-\cos \alpha_{\mathrm{ICM}, \max }(\theta)\right]$

is the factor accounting for magnetic effects. This completes the calculation of the contribution to the ionization of CRs coming from the upper side of the cloud.

A CR propagating along a field line and reaching a polar angle $\theta$ coming from the lower side of the cloud has passed through a column density

$N_{0}(\pi / 2)+\left[N_{0}(\pi / 2)-N_{0}(\theta)\right]=2 N_{0}(\pi / 2)-N_{0}(\theta)$,

where $N_{0}(\pi / 2)$ is the cloud's column density at the midplane. Thus, the contribution of CRs coming from the lower side of the cloud (denoted by a subscript -) to the ionization rate is

$\zeta_{-}^{\mathrm{H}_{2}}(\theta)=\varphi_{-}(\theta) \zeta_{0}^{\mathrm{H}_{2}}\left[2 N_{0}(\pi / 2)-N_{0}(\theta)\right]$,

where $\varphi_{-}(\theta)$ is

$\varphi_{-}(\theta)=\frac{\chi(\theta)}{2}\left(1-\cos \alpha_{\mathrm{cr}}\right)$

The total CR ionization rate is then given by

$\zeta^{\mathrm{H}_{2}}(\theta)=\zeta_{+}^{\mathrm{H}_{2}}(\theta)+\zeta_{-}^{\mathrm{H}_{2}}(\theta)$.

Figure 5 shows $\zeta^{\mathrm{H}_{2}}(\theta)$ including the effects of magnetic focusing and mirroring, compared to the $\mathrm{CR}$ ionization rate evaluated without considering the magnetic field. The curves refer to the four combinations of the two protons and electron spectra assumed in this work (see the paragraph following Eq. (13)). It is

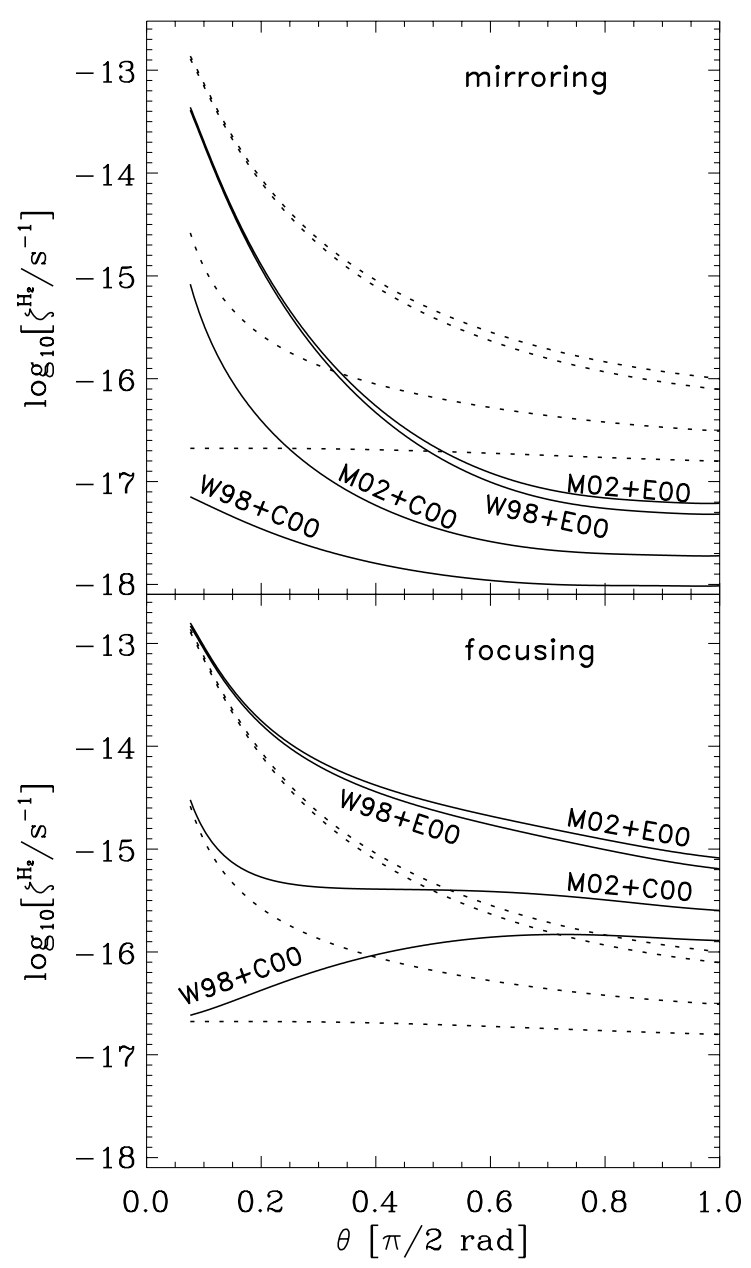

Fig. 5. Effects of magnetic mirroring (upper panel, solid lines) and focusing (lower panel, solid lines) on the CR ionization rate as function of the polar angle. Dotted lines represent the case without considering the magnetic field influence, from P09. Labels refer to the CR proton and electron spectra used in P09 in the non-magnetic field case (proton spectra: Webber 1998, W98; Moskalenko et al. 2002, M02; electron spectra: Strong et al. 2000, C00 and E00).

evident that the two processes have opposite effects of comparable magnitude on the ionization rate, both becoming more and more important approaching the core's midplane where the field is stronger. In Fig. 6 we compare the values of $\zeta^{\mathrm{H}_{2}}$ computed with and without magnetic effects as function of the column density (measured along field lines) in the core. The quantity $\mathcal{R}$, defined as the ratio of the rates obtained in the two cases, is shown in the lower panel of the figure. Clearly, magnetic mirroring always reduces the $\mathrm{CR}$ ionization rate more than magnetic focusing can increase it, the total effect being a net reduction of $\zeta^{\mathrm{H}_{2}}$ by a factor between 2 and 3 . Notice that the maximum effect of the magnetic on $\zeta^{\mathrm{H}_{2}}$ is not obtained on the core's midplane, where the field is stronger, but at an intermediate position corresponding to column densities $10^{21}-10^{22} \mathrm{~cm}^{-2}$. In fact, in the core's midplane $\varphi_{+}(\pi / 2)=\varphi_{-}(\pi / 2)=\chi(\pi / 2)\left(1-\cos \alpha_{\mathrm{cr}}\right) / 2$, and, using Eq. (3), one has

$\mathcal{R}(\pi / 2)=\chi(\pi / 2)\left(1-\cos \alpha_{\mathrm{cr}}\right)=\chi(\pi / 2)\left(1-\sqrt{1-\frac{1}{\chi(\pi / 2)}}\right)$

independent on the assumed CR spectrum, a result also obtained by Desch et al. (2004). For the reference flux tube enclosing $1 M_{\odot}, \mathcal{R}(\pi / 2)=0.516$. 


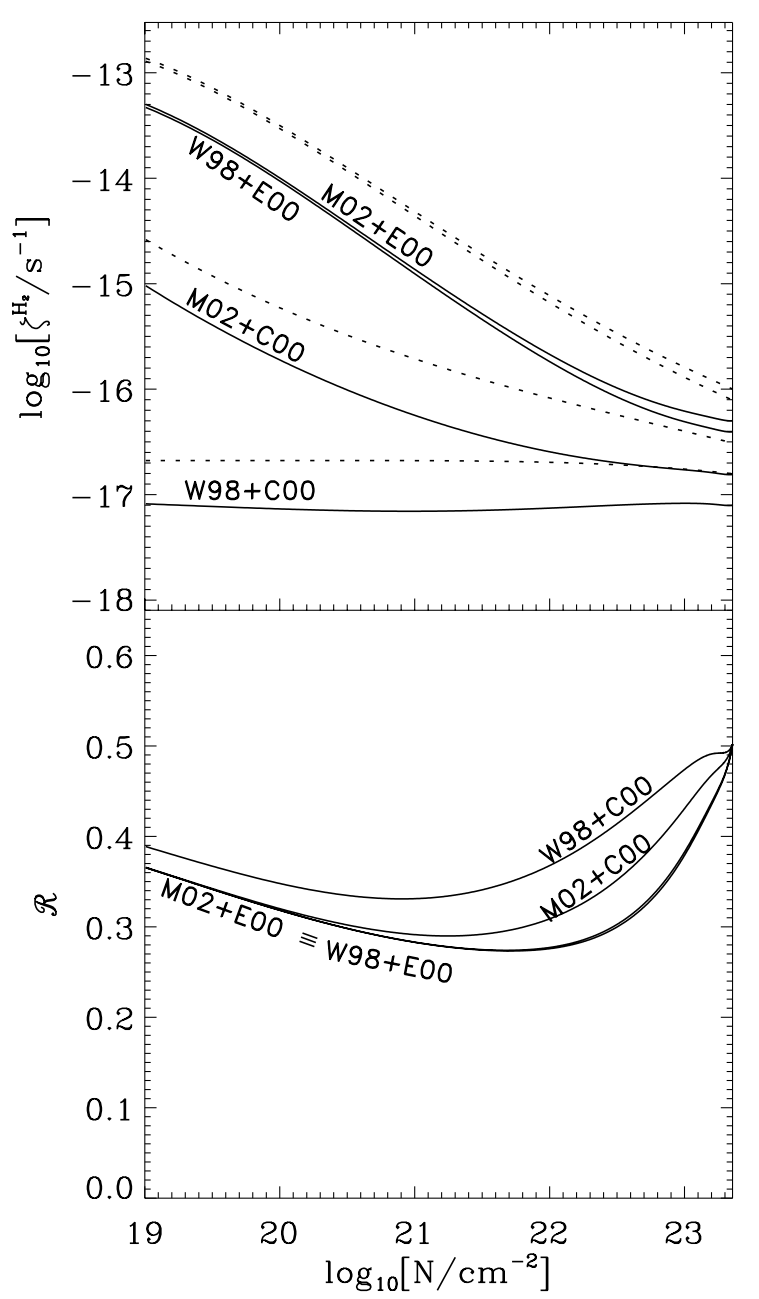

Fig. 6. Comparison between the $\mathrm{CR}$ ionization rate with and without the effects of magnetic field (solid and dotted lines, respectively) as function of the column density (upper panel); ratio between the ionization rates in the magnetic and non-magnetic case (lower panel). The curves are labeled as in Fig. 5.

\section{Dependence of $\zeta^{\mathrm{H}_{2}}$ on the mass and magnetization of the core}

\subsection{Varying the enclosed mass $M(\Phi)$}

In the previous section we have considered $\mathrm{CR}$ propagating along the field lines of a flux tube enclosing $1 M_{\odot}$. In Table 1 we show the variation of the relevant quantities of the problem as function of the enclosed mass for our reference core with $\lambda=2.66$. Flux tubes enclosing smaller masses intersect the midplane at smaller $r_{\mathrm{eq}}$ and are characterized by larger values of the magnetic field and density (see Fig. 2). In particular, in our model the field strength and the density increase inward as $|\boldsymbol{B}| \propto r^{-1} \propto M(\Phi)^{-1}$ and $\rho \propto r^{-2} \propto M(\Phi)^{-2}$, respectively (see Eqs. (10) and (8)). As a consequence, focusing becomes more important, since $\chi$ increases, but also mirroring becomes more severe, since $\alpha_{\mathrm{cr}}$ decreases. The net effect is a stronger reduction of $\zeta^{\mathrm{H}_{2}}$ in the innermost regions of the core as compared to the envelope (see Table 1). As the field strength increases approaching the central singularity of the model, $\chi \rightarrow \infty$ and the reduction factor of the $\mathrm{CR}$ ionization rate in the core's midplane approaches the asymptotic value $\mathcal{R}(\pi / 2)=1 / 2$.

Conversely, for flux tubes enclosing larger masses, the field strength approaches the ICM value, and the density decreases
Table 1. Values of the parameters described in the text as function of the mass, $M(\Phi)$, contained within flux tube $\Phi$.

\begin{tabular}{cccccc}
\hline \hline $\begin{array}{c}M(\Phi) \\
\left(M_{\odot}\right)\end{array}$ & $\begin{array}{c}r_{\mathrm{eq}} \\
(\mathrm{pc})\end{array}$ & $\begin{array}{c}N_{0}(\pi / 2) \\
\left(10^{23} \mathrm{~cm}^{-2}\right)\end{array}$ & $\alpha_{\mathrm{cr}}$ & $\chi(\pi / 2)$ & $\mathcal{R}(\pi / 2)$ \\
\hline 0.5 & 0.018 & 4.54 & $14.8^{\circ}$ & 15.347 & 0.508 \\
1 & 0.036 & 2.27 & $20.5^{\circ}$ & 8.174 & 0.516 \\
5 & 0.180 & 0.45 & $39.9^{\circ}$ & 2.435 & 0.566 \\
10 & 0.360 & 0.23 & $49.7^{\circ}$ & 1.717 & 0.608 \\
50 & 1.802 & 0.05 & $69.3^{\circ}$ & 1.143 & 0.739 \\
100 & 3.604 & 0.02 & $75.0^{\circ}$ & 1.072 & 0.794 \\
\hline
\end{tabular}

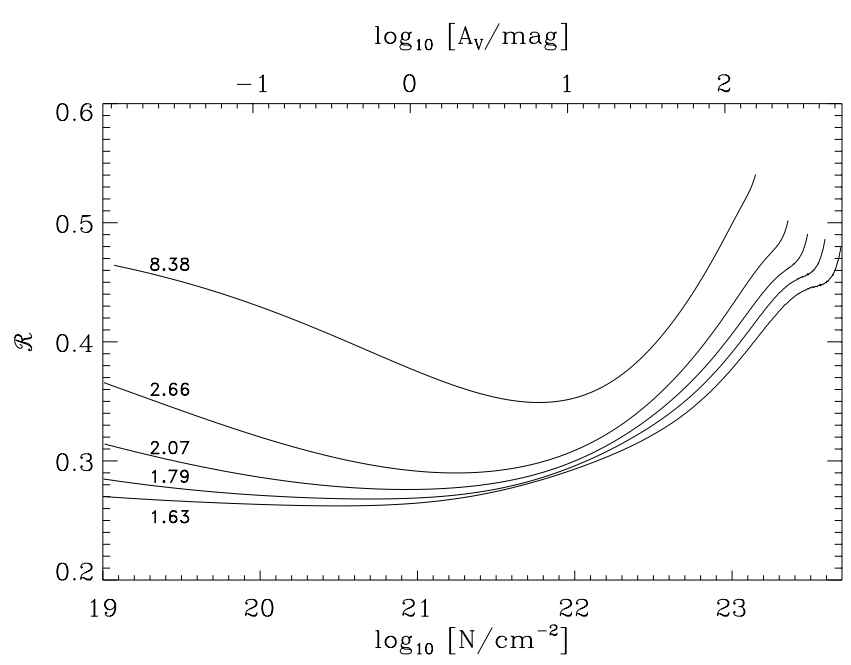

Fig. 7. Ratio $\mathcal{R}$ between the $\mathrm{CR}$ ionization rates in the magnetic and non-magnetic case for the case of $\mathrm{M} 02+\mathrm{C} 00$ spectrum (see Fig. 5 for the labels). The curves are computed for a flux tube containing $1 M_{\odot}$ and for different values of $\lambda=8.38,2.66,2.07,1.79,1.63$. The upper scale shows the extinction through the cloud obtained from $A_{V} / \mathrm{mag}=N / 10^{21} \mathrm{~cm}^{-2}$.

to zero. Therefore both focusing and mirroring become weaker, and $\alpha_{\mathrm{cr}}$ approaches $90^{\circ}$, as shown by Table 1 . As expected, for increasing values of $M(\Phi), \zeta^{\mathrm{H}_{2}}$ approches the value of the non-magnetic case, because the magnetic field strength decreases away from the center of the core, approaching the ICM value.

\subsection{Varying the mass-to-flux ratio $\lambda$}

In this section we explore the effects of the variation of the core's mass-to-flux ratio $\lambda$ on $\zeta^{\mathrm{H}_{2}}$, for a flux tube containing a fixed mass of $1 M_{\odot}$. For cores with strong magnetic support (lower values of $\lambda$ ), the equatorial squeezing of the field lines is stronger, and the lines reach more internal regions of the core where the density is higher. As a consequence, $\alpha_{\mathrm{cr}}$ decreases, the mirroring effect becomes stronger, and a smaller fraction of CRs can penetrate the cloud. In Fig. 7 we show the ratio $\mathcal{R}$ as a function of column density for decreasing values of $\lambda$. For simplicity, we have considered only the combination of the proton spectrum M02 and the electron spectrum C00 (the results obtained with the other spectra are similar). As the figure shows, the reduction of $\zeta^{\mathrm{H}_{2}}$ is larger in cores with larger magnetic support, due to the increase in the field strength and concentration of field lines. The reduction is a factor $\sim 4$ for the outer regions of cores with $\lambda=1.63$, the lowest value of mass-to-flux ratio considered in our models. 
For $\lambda \rightarrow 1$, the density distribution becomes more and more flattened, the core assumes the shape of a thin disk, and the column density from the ICM to the core's midplane becomes larger. In this limiting case, the magnetic field strength and the column density increase as $\left(\lambda^{2}-1\right)^{-2}$. For these magnetically dominated disk-like configurations, the reduction of the $\mathrm{CR}$ ionization rate approaches the asymptotic value $1 / 2$.

\section{Conclusions}

We have determined the $\mathrm{CR}$ ionization rate $\zeta^{\mathrm{H}_{2}}$ in a magnetically supported molecular core, and compared the results with the case of non-magnetized density distribution with the same column density. We have considered only the large-scale magnetic field threading the core because, according to previous investigations, CR scattering by magnetic fluctuation on the scale of the Larmor radius of the particle should not be important for CRs in the energy range $100 \mathrm{MeV}-1 \mathrm{GeV}$, responsible for the bulk of the ionization and heating in molecular clouds.

Assuming the hourglass magnetic field profile and the density distribution of a model of magnetically supported cloud core, we computed $\zeta^{\mathrm{H}_{2}}$ as function of position in the core, for different values of the intercloud CR flux and composition, taking into account the energy loss processes considered in the previous work of P09 and following the CR propagation along magnetic field lines. The relatively weak dependence of the reduction of the CR ionization rate on the mass enclosed in a flux tube (basically a logarithmic dependence, see Table 1 ), and on the mass-toflux ratio of the cloud core $(\lambda \approx 2$ on average for most cores, see Troland \& Crutcher 2008), lends some confidence on the general validity of our results beyond the specific cloud model adopted here. The method described in this paper can be applied however to any axisymmetric cloud model, provided the input quantities $B$ and $\rho$ along a given flux tube are specified as function of the polar angle or vertical cylindrical coordinate, as in Fig. 2.

Our results show that $\zeta^{\mathrm{H}_{2}}$ in a magnetized core is always reduced with respect to its non-magnetic value, by a factor depending on the core's mass-to-flux ratio (determining the field strength) and the amount of mass contained in the flux tube considered. In general, for a flux tube enclosing about $1 M_{\odot}$, and for a core with mass-to-flux ratio $\lambda=2.66$, the CR ionization rate is reduced by a factor of $\sim 3$ over most of the core, and by a factor of $\sim 2$ near the core's midplane. The reduction is less severe for flux tubes enclosing larger masses and for larger values of the core's mass-to-flux ratio, because of the near uniformity of the magnetic field in these cases. For cores with mass-to-flux ratio $\lambda \approx 1.6$ or lower, the magnetic field can decrease the CR ionization rate by a factor of $\sim 4$ over most of the core's envelope.
Thus, the values of $\zeta^{\mathrm{H}_{2}}$ derived by Caselli et al. (1998), Williams et al. (1998), Maret \& Bergin (2007) for dense cores and globules, probably underestimate the "external" (i.e. intercloud) $\mathrm{CR}$ ionization rate by a factor of $\sim 3-4$, thus alleviating the discrepancy with measurements of $\zeta^{\mathrm{H}_{2}}$ in diffuse clouds. In this respect, it is interesting to notice that the best overall fit with the chemical abundances of the low-mass core TMC- 1 is obtained when the $\mathrm{CR}$ ionization rate is reduced by a factor $\sim 5$ with respect to the standard value $\zeta^{\mathrm{H}_{2}} \approx 10^{-17} \mathrm{~s}^{-1}$, together with the rate of removal of atomic hydrogen from the gas phase (Flower et al. 2007). However, the observed large scatter in the values of $\zeta^{\mathrm{H}_{2}}$ of dense clouds found by Caselli et al. (1998), if real, cannot be attributed to effects of the mean field or to variations in the mass-to-flux ratio of the cores.

Acknowledgements. This work was partially supported by the Marie-Curie Research Training Network "Constellation” (MRTN-CT-2006-035890).

\section{References}

Caselli, P., Walmsley, C. M., Terzieva, R., \& Herbst, E. 1998, ApJ, 499, 234 Cesarsky, C. J., \& Völk, H. J. 1978, A\&A, 70, 367

Chandran, B. D. G. 2000, ApJ, 529, 513

Dalgarno, A. 2006, Publ. Nat. Acad. Sci., 103, 12269

Desch, S. J., Connolly, H. C., Jr., \& Srinivasan, G. 2004, ApJ, 602, 528

Flower, D. R., Pineau Des Forêts, G., \& Walmsley, C. M. 2007, A\&A, 474, 923 Hasegawa, T. I., \& Herbst, E. 1993, MNRAS, 263, 589

Hayakawa, S., Nishimura, S., \& Takayanagi, T. 1961, PASJ, 13, 184

Gabici, S., Aharonian, F. A., \& Blasi, P. 2007, Ap\&SS, 309, 365

Galli, D., Lizano, S., Li, Z.-Y., Adams, F. C., \& Shu, F. H. 1999, ApJ, 521, 630

Girart, J. M., Rao, R., \& Marrone, D. P. 2006, Science, 5788, 812

Glassgold, A. E., \& Langer, W. D. 1973, ApJ, 186, 859

Gonçalves, J., Galli, D., \& Girart, J. M. 2008, A\&A, 490, L39

Kulsrud, R. M. 2005, Plasma physics for astrophysics (Princeton University Press)

Kulsrud, R., \& Pearce, W. P. 1969, ApJ, 156, 445

Li, Z.-Y., \& Shu, F. H. 1996, ApJ, 472, 211

Maret, S., \& Bergin, E. A. 2007, ApJ, 664, 956

Moskalenko, I. V., Strong, A. W., Ormes, J. F., \& Potgieter, M. S. 2002, ApJ, 565,280

Padoan, P., \& Scalo, J. 2005, ApJ, 624, L97

Padovani, M., Galli, D., \& Glassgold, A. E. 2009, A\&A, 501, 619 (P09)

Pinto, C., Galli, D., \& Bacciotti, F. 2008, A\&A, 484, 1

Skilling, J., \& Strong, A. W. 1976, A\&A, 53, 253

Spitzer, L., \& Tomasko, M. G. 1968, ApJ, 152, 971

Strong, A. W., Moskalenko, I. V., \& Reimer, O. 2000, ApJ, 537, 763

Tang, Y.-W., Ho, P. T. P., Koch, P. M., et al. 2009, ApJ, 700, 251

Troland, T. H., \& Crutcher, R. M. 2008, ApJ, 680, 457

Wakelam, V., Herbst, E., Selsis, F., \& Massacrier, G. 2006, A\&A, 459, 813

Williams, J. P., Bergin, E. A., Caselli, P., Myers, P. C., \& Plume, R. 1998, ApJ, 503,689

Webber, W. R. 1998, ApJ, 506, 329 